\title{
Universia: Um Aplicativo Colaborativo de Suporte a Grupos Acadêmicos
}

\author{
Lajos Onodi, Ingrid Nascimento, Bruno Gadelha \\ Instituto de Computação - Universidade Federal do Amazonas (UFAM) \\ CEP 69.077-000 - Manaus - AM - Brasil \\ lajosneto@gmail.com, \{inc, bruno\}@icomp.ufam.edu.br
}

\begin{abstract}
This paper presents the Universia, a collaborative application for mobile devices that aims to support academic content sharing to spontaneously formed groups. As a differential, the application allows members themselves manage groups and share content without direct interference from a teacher. The application is presented as one of the results of a research that aims to understand the spontaneous formation of groups of students in an academic context, conducted according to the design science research approach. The application was evaluated with respect to their usability and user experience.
\end{abstract}

Resumo. Este artigo apresenta o Universia, um aplicativo colaborativo para dispositivos móveis que tem por objetivo dar suporte ao compartilhamento de conteúdo acadêmico para grupos formados espontaneamente. Como diferencial, o aplicativo possibilita que os próprios membros do grupo gerenciem e compartilhem conteúdo, sem a interferência direta de um professor. $O$ aplicativo se apresenta como um dos resultados de uma pesquisa que visa compreender a formação espontânea de grupos de alunos no ambiente universitário, desenvolvida segundo a design science research. $O$ aplicativo foi avaliado com relação à sua usabilidade e experiência do usuário.

\section{Introdução}

É comum no ambiente acadêmico observar a formação de grupos. Esses grupos podem ser de estudos, grupos de pesquisa, grupos informais de amigos e grupos multiobjetivos que podem agregar indivíduos com múltiplos interesses. Esses grupos são motivados pela necessidade de colaborar para atingir um determinado objetivo. Vivacqua \& Garcia (2011) destacam que os grupos podem ser formados por duas maneiras: por iniciativa de uma entidade externa ou de forma espontânea.

No contexto acadêmico, muitos grupos são criados por iniciativa de uma entidade externa: o professor. Nesse caso, os grupos são induzidos pelo professor que pode estabelecer regras para essa formação de acordo com seus objetivos pedagógicos. Porém, muitos outros grupos emergem espontaneamente tanto entre colegas de turma quanto grupos que são transversais às formatações formais de turmas e cursos de uma universidade. Tais grupos podem, inclusive, tornar-se comunidades espontâneas. Nejma et. al. (2014) definem comunidades espontâneas como um grupo espontâneo de indivíduos que compartilham um mesmo interesse ou propósito relacionado a uma situação circunstancial e relativo a uma localização geográfica. 
V Congresso Brasileiro de Informática na Educação (CBIE 2016)

Anais do XXVII Simpósio Brasileiro de Informática na Educação (SBIE 2016)

Esses últimos são os grupos de interesse dessa pesquisa uma vez que são formados pelos próprios membros e não possuem, a priori, papéis e objetivos bem definidos. Assim, este artigo apresenta o Universia, um aplicativo que tem por objetivo dar suporte à criação e compartilhamento de conteúdo a esse tipo de grupo. As funcionalidades previstas no aplicativo foram desenvolvidas à luz do Modelo 3C de Colaboração conforme Fuks et. al (2007).

O Universia apresenta-se como resultado parcial de uma pesquisa mais ampla que busca entender os critérios de formação e ciclo de vida desses grupos espontâneos. A pesquisa está sendo conduzida através de um método que implementa a abordagem da Design Science Research, descrito em Dresch et.al. (2015). O aplicativo foi avaliado segundo os critérios de usabilidade e experiência do usuário (UX).

O artigo está organizado da seguinte forma: a Seção 2 apresenta os conceitos relacionados à pesquisa apresentada; a seguir a metodologia de pesquisa é descrita na Seção 3; a Seção 4 apresenta o aplicativo Universia; o aplicativo foi submetido a uma avaliação de usabilidade e experiência do usuário (UX) e tal avaliação, em conjunto com a análise dos resultados, é descrita na Seção 5; por fim, na Seção 6, são apresentadas as conclusões do trabalho.

\section{Fundamentação Teórica}

Esta seção aborda os principais conceitos relacionados à pesquisa e ao desenvolvimento do aplicativo apresentado neste artigo que são: grupos de aprendizagem, colaboração e Modelo 3C de Colaboração.

\subsection{Grupos de Aprendizagem}

Segundo Santos, Castro e Castro (2007), um grupo é um pequeno conjunto de indivíduos que se reúnem por meio de uma determinada peculiaridade ou objetivo. Para que esses indivíduos reúnam-se em grupos, alguns aspectos devem ser considerados como: abordagens, critérios, características e necessidades desses indivíduos. No caso dos grupos de apoio às atividades acadêmicas os indivíduos, que podem ser estudantes, professores e tutores, compartilham as necessidades acadêmicas de aprendizagem. Esses agrupamentos podem ser relevantes na aprendizagem individual e na colaborativa.

Para Dillenbourg et al. (1996), a aprendizagem colaborativa ocorre quando um grupo de pessoas se motivam em aprender algo juntas, o que fomenta a criação de grupos. O agrupamento dos indivíduos no contexto acadêmico pode ser realizado, de acordo com Ounnas, Davis e Millard (2007), de três maneiras: randômico, autoselecionado e selecionado. Os grupos randômicos são propostos pelo professor, que agrupa os estudantes sem nenhum critério definido. Grupos auto-selecionados são aqueles em que os alunos escolhem o grupo ao qual querem pertencer. Grupo selecionado acontece quando o professor define os critérios específicos para a formação dos grupos.

Citadin, Kemczinski e Matos (2014) classificam os grupos em homogêneos, heterogêneos e ambos. Em um grupo homogêneo, seus membros apresentam características semelhantes. Em um grupo heterogêneo, seus membros apresentam características diferentes. O caso "ambos" acontece quando o contexto de formação de grupos possibilita a formação de grupos homogêneos ou heterogêneos. 
Para esta pesquisa, os grupos de interesse são auto-selecionados e heterogêneos, uma vez que se quer observar como tais grupos são livremente criados e mantidos pelos estudantes. Outro ponto de interesse é o ciclo de vida dos grupos criados, onde as informações de duração dos grupos e motivo para a existência deles são investigados. Esses grupos auto-selecionados e heterogêneos que surgem por iniciativa livre dos indivíduos são considerados nesse artigo como grupos espontâneos. Os membros de tais grupos espontâneos colaboram entre si e, no contexto acadêmico, podem vir a formar comunidades espontâneas de aprendizagem. A colaboração entre os membros desses grupos é analisada segundo o Modelo 3C de Colaboração.

\subsection{Colaboração e Modelo 3C de Colaboração}

A colaboração nesta pesquisa é analisada de acordo com o Modelo 3C ilustrado pela Figura 1, que considera que esta é alcançada através da interação entre a comunicação, coordenação e cooperação. Durante a comunicação ocorre uma troca de mensagens objetivando futura ação comum. Coordenação trata das pessoas e suas interdependências necessárias para o comprimento do plano de ação acordado. Cooperação compreende as ações tomadas pelos membros do grupo no espaço compartilhado.

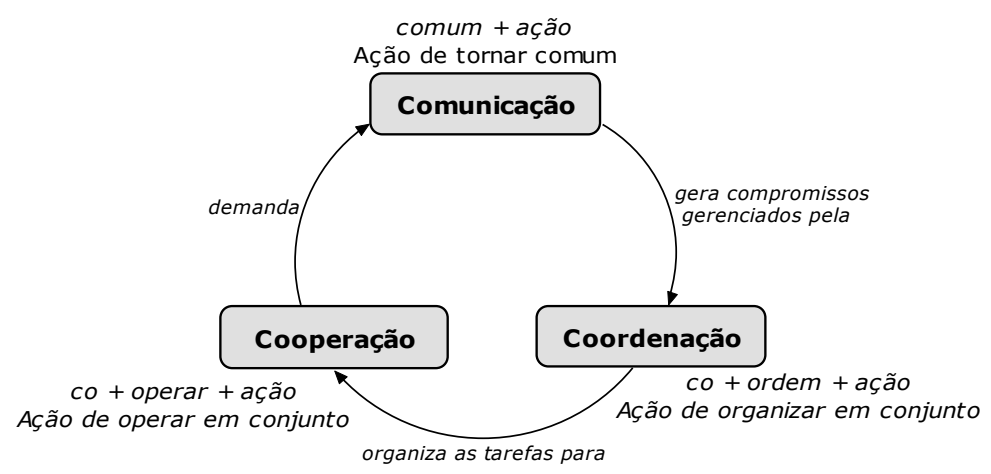

Figura 1. Modelo 3C de Colaboração. Fonte: Pimentel et. al. (2008)

Para dar suporte à comunicação, Fuks et.al. (2007) afirmam que o projetista das ferramentas de comunicação define os elementos de comunicação que, por sua vez, definem o canal de comunicação entre os interlocutores de acordo com o propósito, dinâmica, tempo e espaço. De acordo com as necessidades do grupo, aspectos como privacidade e sobrecarga de informação devem ser levados em consideração.

Se o objetivo for coordenar pessoas, o foco da coordenação deve ser nas ferramentas que provêm agenda e contexto. Coordenar recursos está relacionado ao espaço compartilhado, onde as ações acontecem. Coordenar tarefas consiste em gerenciar interdependências entre tarefas que devem ser executadas para atingir o objetivo do grupo. Então, o projetista deve considerar esses diferentes aspectos da coordenação ao projetar ferramentas para suportá-la.

Para dar suporte à cooperação, o projetista deve configurar o espaço compartilhado onde as ações irão acontecer. Um conjunto de ferramentas para armazenamento e manutenção de artefatos (documentos, planilhas, apresentações e outros) deve ser oferecido. 
V Congresso Brasileiro de Informática na Educação (CBIE 2016)

Anais do XXVII Simpósio Brasileiro de Informática na Educação (SBIE 2016)

O Modelo 3C de Colaboração foi utilizado nesta pesquisa como norte para a concepção das funcionalidades para dar suporte à colaboração entre os integrantes dos grupos espontâneos no aplicativo proposto.

\section{Metodologia}

O aplicativo desenvolvido e descrito neste artigo surgiu como resultado parcial de uma pesquisa mais ampla que tem por objetivo estudar os grupos acadêmicos de formação espontânea. A metodologia utilizada na pesquisa é a Design Science Research (DSR). De acordo com Dresch et. al. (2015), a DSR consiste em uma forma de produção de conhecimento científico que envolve o desenvolvimento de uma inovação, com a intenção resolver problemas do mundo real e, ao mesmo tempo, fazer uma contribuição científica de caráter prescritivo. De forma bem resumida, a DSR prevê as seguintes atividades: definição do problema a ser resolvido; proposta de artefato para solução do problema; desenvolvimento e avaliação do artefato; conclusões e divulgação dos resultados.

O problema considerado na pesquisa consiste em analisar o comportamento dos estudantes no cenário acadêmico afim de entender a dinâmica utilizada por eles para formação de grupos de estudo/trabalho na universidade. Entende-se que esses grupos são formados espontaneamente entre os estudantes. Assim, pretende-se entender os critérios de formação desses grupos, seus objetivos, formas de trabalho e duração dos grupos.

$\mathrm{O}$ artefato proposto para estudar o problema levantado consiste no aplicativo Universia que será descrito em detalhes na Seção 4. O objetivo do aplicativo é dar suporte tecnológico à formação e gerência desses grupos, bem como dar suporte à colaboração entre seus membros através de funcionalidades com propósitos de coordenação, cooperação e comunicação definidos pelo Modelo 3C de Colaboração. $\mathrm{O}$ Universia foi desenvolvido para a plataforma Android dado que é a plataforma mais utilizada atualmente por usuários de smartphones, além de ser uma plataforma aberta e livre.

Para avaliar o artefato proposto, realizou-se uma inspeção de usabilidade e experiência do usuário (UX - User Experience). Essa inspeção utilizou uma técnica proposta por Nascimento et. al. (2016) chamada Userbility que tem como objetivo integrar a avaliação de usabilidade com a avaliação de UX para auxiliar profissionais não especialistas em IHC (Interação Humano-Computador). Essa técnica contém: doze heurísticas de usabilidade para aplicações móveis com itens de verificação; duas questões de UX com exemplos para cada heurística de usabilidade e; um item relacionado à satisfação dos avaliadores, representado por um rosto humano e uma descrição. Essa avaliação é descrita em detalhes na Seção 5.

\section{O Aplicativo Universia}

O Universia é um aplicativo para plataformas móveis concebido com o objetivo de ser uma plataforma colaborativa onde estudantes fossem capazes de realizar $o$ compartilhamento de conteúdos relacionados as diversas disciplinas e grupos de estudos existentes dentro da universidade. Diferente das opções já existentes, como por exemplo ambientes virtuais de aprendizagem como o Moodle (2016), o Universia não traz o professor como a figura principal dos grupos. Esse papel é exercido pelos próprios 
V Congresso Brasileiro de Informática na Educação (CBIE 2016)

Anais do XXVII Simpósio Brasileiro de Informática na Educação (SBIE 2016)

estudantes que realizam a tarefa de administrar e fornecer o conteúdo dos grupos onde estão inseridos.

De acordo com o Modelo 3C de Colaboração adotado, o aplicativo tem o propósito de "Coordenação" dado que sua principal finalidade é a gerência de grupos de estudantes de forma espontânea no ambiente acadêmico. Sua principal funcionalidade é a criação de grupos. Os estudantes podem criar grupos de disciplinas, grupos de estudos ou grupos de pesquisa, os identificando com informações como: nome do grupo, código, professor responsável ou apelido do grupo/disciplina. Assim como criar grupos, os usuários podem se inscrever/participar de qualquer grupo cadastrado. A partir do momento em que um estudante participa de um determinado grupo, ele passa a ter acesso a todo o conteúdo enviado por todos os outros membros deste grupo. A Figura 2 ilustra as funcionalidades relacionadas à manutenção de grupos que são: adicionar novo grupo, listagem de grupos e detalhes dos grupos com suas ferramentas de adicionar arquivos, eventos e fórum de discussão.

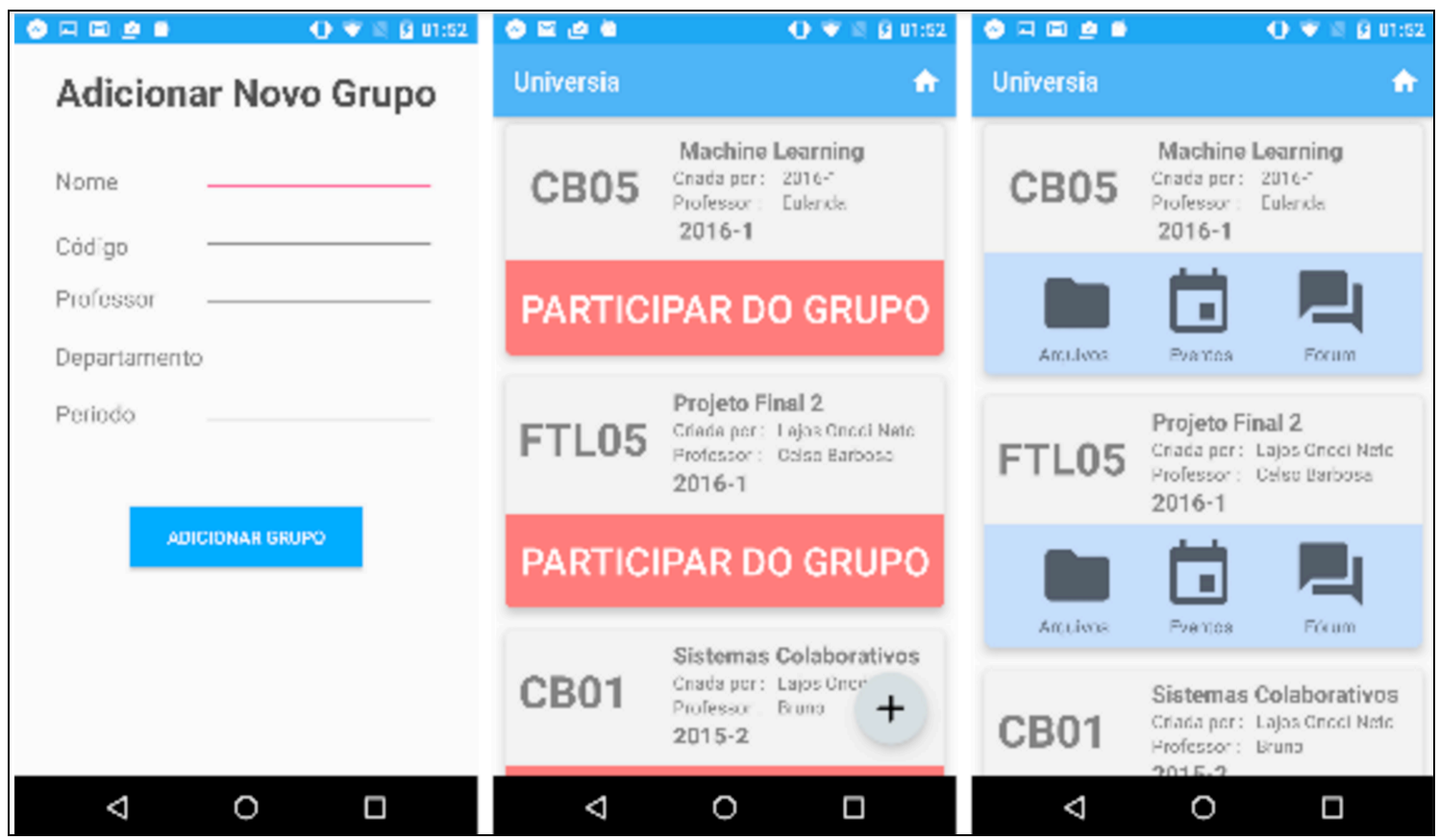

Figura 2. Telas de criação e listagem de grupos no Universia

O Universia disponibiliza três funcionalidades para dar suporte ao compartilhamento de conteúdo entre os estudantes participantes dos grupos. A primeira funcionalidade que, de acordo com o Modelo 3C de Colaboração, tem por propósito a coordenação das atividades dos grupos possibilita aos estudantes agendarem no calendário do grupo as datas dos próximos eventos importantes, informando assim a todos os outros participantes deste grupo. Para facilitar o acesso aos agendamentos de diferentes grupos, o aplicativo conta com um resumo de calendários. Se, por exemplo, um determinado estudante participa de 10 grupos que possuem diversos eventos cadastrados, a verificação manual de cada calendário de eventos seria uma atividade que demandaria um esforço significativo. Assim, o Universia apresenta ao estudante uma lista contendo todos os eventos de todos os seus grupos, ordenados cronologicamente. 
V Congresso Brasileiro de Informática na Educação (CBIE 2016)

Anais do XXVII Simpósio Brasileiro de Informática na Educação (SBIE 2016)

A segunda funcionalidade do aplicativo tem o propósito de cooperação segundo o Modelo 3C de Colaboração. Esta funcionalidade consiste no compartilhamento de URLs de arquivos armazenados em serviços de nuvem como Dropbox e Google Drive. Os usuários podem enviar a URL de arquivos como PDF, slides e fotos. Essa é uma ferramenta muito importante uma vez que se algum estudante faltar alguma das aulas, outros membros podem enviar para o aplicativo fotos de suas anotações por exemplo, facilitando assim a resolução de muitas situações.

A terceira funcionalidade é o envio de mensagens para os grupos. De acordo com o Modelo 3C de Colaboração, esta funcionalidade tem o propósito de comunicação. $\mathrm{O}$ envio de mensagens funciona como um fórum com mensagens organizadas linearmente ou um mural de avisos, onde os usuários podem deixar mensagens para todos os outros usuários. Diferente de um fórum de discussão tradicional, onde as mensagens são hierarquizadas, as mensagens desta ferramenta são exibidas de forma cronológica, onde a última mensagem enviada é a primeira a ser visualizada, o que possibilita ter acesso mais fácil às mensagens com notícias mais recentes dos grupos.

A Figura 3 ilustra as funcionalidades descritas. A primeira tela consiste na funcionalidade de calendário de eventos dos grupos. A segunda apresenta uma lista de arquivos compartilhados com os membros de um grupo. Por fim, a terceira tela apresenta a funcionalidade fórum com algumas mensagens trocadas entre estudantes participantes dos grupos.

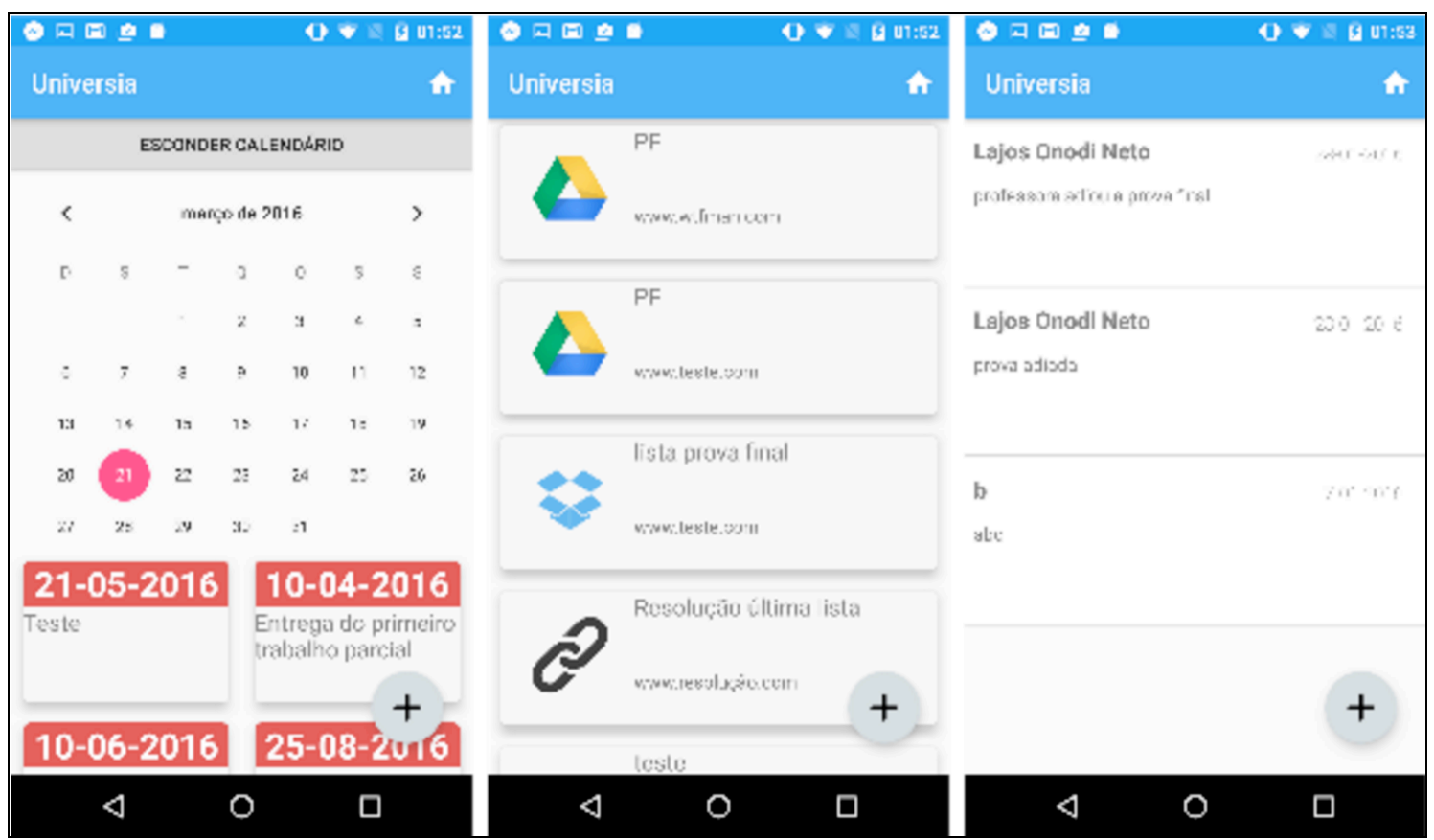

Figura 3. Telas das funcionalidades internas de cada grupo.

\section{Avaliação: Inspeção da Usabilidade e Experiência do Usuário (UX)}

Com o objetivo de avaliar o aplicativo Universia antes de ser transferi-lo para indústria, foi realizado um estudo utilizando a técnica Userbility para avaliar a UX e usabilidade 
V Congresso Brasileiro de Informática na Educação (CBIE 2016)

Anais do XXVII Simpósio Brasileiro de Informática na Educação (SBIE 2016)

da aplicação. A seguir são apresentadas as etapas realizadas no estudo do aplicativo Universia.

\subsection{Preparação}

Inicialmente foi realizado um planejamento das atividades, visando identificar como seria aplicado o estudo do aplicativo. Nessa etapa foram definidos os seguintes recursos:

- Participantes: Os participantes do estudo foram 03 estudantes pós-graduação do grupo de Usabilidade e Engenharia de Software (USES) da Universidade Federal do Amazonas (UFAM).

- Treinamento: Foi realizado um treinamento introduzindo os conceitos de UX e usabilidade e um treinamento da técnica Userbility. No entanto, os participantes já eram experientes com inspeção de usabilidade e com a técnica Userbility, antes do estudo ministrado e dos treinamentos.

- Contexto: Foi introduzido o escopo do aplicativo Universia, para que os participantes pudessem entender o contexto da aplicação. O escopo foi: "O aplicativo Universia oferece aos alunos da universidade uma ferramenta que possibilita a criação e participação de grupos com um interesse em comum, como disciplinas, projetos da universidade ou projetos pessoais. Os alunos podem compartilhar conteúdos, eventos ou mensagens nos grupos com outros alunos. Por exemplo, Joana é uma aluna da UFAM que estuda no IComp. Ela gostaria de um aplicativo onde pudesse participar de grupos de estudos para as disciplinas, trocar mensagens e compartilhar conteúdos com outros alunos".

- Atividade definidas: Cada participante realizou seis atividades principais definidas no aplicativo. As atividades foram: (a) Criar uma conta e fazer login; (b) Criar um grupo; (c) Participar de um grupo; (d) Escolher um grupo e criar um evento para esse grupo; (e) Enviar uma mensagem ao grupo; (f) Enviar um link de um arquivo para esse grupo.

\subsection{Execução}

O processo de inspeção foi conduzindo usando a técnica Userbility para avaliar a aplicação por todos os participantes. Durante a avaliação, os participantes receberam celulares para avaliar a aplicação e cada participante recebeu o contexto e uma lista de tarefas que realizaram na aplicação individualmente.

Após a realização das tarefas, cada participante reportou sua avaliação. As discrepâncias (problemas de usabilidade e UX possíveis indicados por um participante) foram agrupadas em uma única lista. Nessa lista, removemos as discrepâncias duplicadas do mesmo participante antes da discriminação das discrepâncias.

\subsection{Análise dos Dados}

Os resultados quantitativos foram obtidos da após a reunião de discriminação. Foram encontrados 23 defeitos únicos (sem repetição) para serem melhorados na aplicação (5 defeitos cosméticos, 6 defeitos leves, 11 defeitos graves e 1 defeito catastrófico). A Tabela 1 apresenta algumas das discrepâncias encontradas, as sugestões de melhorias dadas pelos participantes do estudo e a severidade do problema. 
V Congresso Brasileiro de Informática na Educação (CBIE 2016)

Anais do XXVII Simpósio Brasileiro de Informática na Educação (SBIE 2016)

O resumo dos resultados por participante que utilizou a Userbility é apresentado na Tabela 2. A Tabela 2 apresenta o número de discrepâncias (ND), o número de falso positivos (NFP), o número de defeitos reais (NRD) e o tempo de inspeção (T) para cada participante.

Tabela 1. Quadro de discrepâncias e sugestões de melhorias (parcial).

\begin{tabular}{|c|c|c|c|c|}
\hline ID & $\begin{array}{c}\text { Descrição do Problema } \\
\text { Encontrado }\end{array}$ & Melhorias & $\begin{array}{l}\text { Problema (P) } \\
\text { ou Falso- } \\
\text { positivo } \\
\text { (FP)? }\end{array}$ & Severidade \\
\hline 1 & $\begin{array}{l}\text { O app não apresenta em } \\
\text { qual opção estamos no } \\
\text { momento. }\end{array}$ & $\begin{array}{l}\text { Cada tela deveria conter a } \\
\text { opção de menu que foi } \\
\text { selecionado "Todos os } \\
\text { grupos, "meus grupos", } \\
\text { "calendário dos grupos". }\end{array}$ & $\mathbf{P}$ & Grave \\
\hline 2 & $\begin{array}{l}\text { Os feedbacks e mensagens } \\
\text { estão muito genéricos, } \\
\text { "mensagem inserida com } \\
\text { sucesso" para todas as } \\
\text { inserções. }\end{array}$ & $\begin{array}{l}\text { As mensagens deveriam } \\
\text { ser específicas para cada } \\
\text { ação do usuário no app. }\end{array}$ & $\mathbf{P}$ & Leve \\
\hline 3 & $\begin{array}{l}\text { Os botões de Menus não } \\
\text { se destacam quando } \\
\text { selecionados. Tudo isso } \\
\text { pode deixar o usuário } \\
\text { perdido. }\end{array}$ & $\begin{array}{l}\text { E os botões poderia } \\
\text { mudar de cor ao serem } \\
\text { selecionados. }\end{array}$ & $\mathbf{P}$ & Leve \\
\hline 5 & $\begin{array}{l}\text { Tem algumas quebras de } \\
\text { palavras com caracteres } \\
\text { especiais, quando as } \\
\text { mesmas possuem acentos. } \\
\text { Alguns erros de português } \\
\text { como "periodo" e "criada } \\
\text { por" e tem mensagens que } \\
\text { no final possuem "!.". }\end{array}$ & $\begin{array}{l}\text { Fazer revisão e verificar } \\
\text { caracteres especiais. }\end{array}$ & $\mathbf{P}$ & Grave \\
\hline 6 & $\begin{array}{l}\text { Me senti limitada pois não } \\
\text { tenho opção de cancelar, } \\
\text { deletar, sair de um grupo, } \\
\text { nem de editar alguma } \\
\text { informação. }\end{array}$ & $\begin{array}{l}\text { Deveria disponibilizar } \\
\text { mais opções para o } \\
\text { usuário que está criando } \\
\text { o grupo, enviando } \\
\text { mensagens, enviando } \\
\text { arquivos, criando eventos } \\
\text { e fazendo login. Como } \\
\text { por exemplo: adicionar, } \\
\text { editar, excluir e cancelar } \\
\text { somente as ações de sua } \\
\text { propriedade. }\end{array}$ & $\mathbf{P}$ & Grave \\
\hline
\end{tabular}


V Congresso Brasileiro de Informática na Educação (CBIE 2016)

Anais do XXVII Simpósio Brasileiro de Informática na Educação (SBIE 2016)

Tabela 2. Resumo dos resultados por participante.

\begin{tabular}{|c|c|c|c|c|}
\hline Participantes & ND & NFP & NRD & T(h) \\
\hline P01 & 20 & 3 & 17 & 1.02 \\
\hline P02 & 12 & 1 & 11 & 0.80 \\
\hline P03 & 22 & 2 & 2 & 1.23 \\
\hline \\
Legenda: ND - Número de Discrepâncias; NFP - Número de Falso Positivos; NRD - \\
Número de Defeitos Reais; T (h) - Tempo em horas.
\end{tabular}

Os resultados qualitativos foram obtidos a partir das respostas sobre a visão geral sobre o aplicativo durante a aplicação da técnica Userbility. Nessa visão geral, foi avaliada a percepção dos participantes sobre a satisfação com o aplicativo, numa escala de 5 pontos (muito insatisfeito, insatisfeito, nem insatisfeito nem satisfeito, satisfeito, muito satisfeito) e o porque dessa avaliação. Os três participantes que avaliaram a aplicação apontaram a opção de satisfeito com a aplicação. As percepções foram:

"Acho o aplicativo muito útil, mas ele deve ser mais atrativo que um WhatsApp para que os alunos o adotem. Eu usaria o aplicativo, mas deve ser visto alguns aspectos de segurança e validação, como por exemplo, não deixar inserir com o mesmo e-mail." (P01);

"No geral, fiquei satisfeito com o aplicativo. O único erro funcional foi 'criar grupo' que não funciona. Os outros erros são detalhes menores que só precisam ser revisados." (P02);

"Achei o aplicativo simples. São necessários apenas alguns ajustes nos padrões dos botões, nas cores do aplicativo, nas mensagens, alguns termos e navegação. Gostei da tela 'Grupos que participo'. A interface do aplicativo poderia seguir esse padrão." (P03).

Além disso, cada participante também forneceu sugestões de melhorias quando apontaram um problema. Essas sugestões de melhorias apontadas pelos participantes servirão para melhorar a aplicação Universia.

\section{Conclusão}

Este artigo apresentou o Universia, um aplicativo colaborativo para dispositivos móveis de suporte à criação e troca de informações em grupos acadêmicos. O objetivo do aplicativo é dar suporte computacional à colaboração de grupos de indivíduos formados espontaneamente. O diferencial desse aplicativo consiste no fato dos grupos serem criados e mantidos completamente por seus membros, ou seja, sem a interferência direta de um professor ou moderador.

O aplicativo foi submetido a uma inspeção de usabilidade e experiência do usuário. Essa inspeção revelou uma série de discrepâncias e defeitos reais. Tais defeitos e discrepâncias foram considerados e uma nova versão foi desenvolvida. A partir de então, um novo estudo faz-se necessário. Esse novo estudo consiste em verificar a aceitação do aplicativo pelo seu público-alvo. Caso o estudo revele que o sistema pode ser adotado, este será implantado e disponibilizado para uso geral na Universidade Federal do Amazonas. 
V Congresso Brasileiro de Informática na Educação (CBIE 2016)

Anais do XXVII Simpósio Brasileiro de Informática na Educação (SBIE 2016)

O aplicativo é o resultado parcial de uma pesquisa mais ampla que tenta compreender como os grupos espontâneos são formados, por quanto tempo persistem, quais os critérios para sua formação, dentre outras informações. Assim, com o uso intensivo do aplicativo espera-se ter uma base de prospecção para investigação de tais questões.

\section{Agradecimentos}

Parte dos resultados apresentados neste artigo foi obtida através de atividades de P\&D do "Programa de Qualificação em Grande Escala em Tecnologias Móveis PROMOBILE”, projeto patrocinado pela Samsung Eletrônica da Amazônia Ltda nos termos da lei federal brasileira ${ }^{\circ} 8.248 / 91$.

\section{Referências}

Citadin, J., Kemczinski, A. e Matos, A. (2014) "Formação de Grupos para Aprendizagem Colaborativa: Um mapeamento sistemático da Literatura", In: XIX Conferência Internacional sobre Informática na Educação, 19,v. 10,p. 46-54.

Dillenbourg, P., Baker, M. Blaye, A. e O' Malley, C. (1996) "The evolution of research on collaborative learning", In: Spada, E., Reiman, P.(eds) Learning in Humans and Machine: Towards an Interdisciplinary Learning Science, p.189-211. Elsevier, Oxford.

Dresch, A.; Lacerda, D. P.; Antunes Jr, J. A. V (2015). Design Science Research: Método de Pesquisa para Avanço da Ciência e Tecnologia. Bookman.

Fuks, H.; Raposo, A.; Gerosa, M.A.; Pimentel, M.; Lucena, C.J.P (2007). The 3C Collaboration Model. The Encyclopedia of E-Collaboration, Ned Kock (org), pp. 637-644.

Moodle. Moodle (2016) - Open-source learning platform. Disponível em: http://moodle.org. Acessado em Março de 2016.

Nascimento, I.; Silva, W.; Gadelha, B.; Conte, T (2016). Userbility: A Technique for the Evaluation of User Experience and Usability on Mobile Applications. In: Lecture Notes in Computer Science. Springer International Publishing, v. 9731, p. 372-383.

Nejma, G. B.; Roose, P.; Dalmau, M.; Gensel, J.; Ghorbali, M. A (2014). Taldea a Spontaneous Community-aware Application in Pervasive Environment. Proceedings of Fourth International conference on selected topics in Mobile and Wireless Networking (MoWNet 2014). Roma, Itália.

Ounnas, A., Davis, H. e Millard, D. (2007) "Towards semantic group formation", In: Proceedings of The 7th IEEE International Conference on Advanced Learning Technologies (ICALT 2007), p. 825-827.

Pimentel, M.; Fuks, H.; Lucena, C.J.P (2008). Um processo de desenvolvimento de sistemas colaborativos baseado no Modelo 3C: RUP-3C-Groupware. Anais do IV SBSI. Rio de Janeiro, Brasil.

Santos, S., Castro, J. e Castro, T. (2007) "Alteração do Modelo de Grupos do Moodle para apoiar a Colaboração", In: Anais do XVIII Simpósio Brasileiro de Informática na Educação (SBIE 2007), 18, p.123-134. Porto Alegre.

Viviacqua, A. S.; Garcia, A. C. B (2011). Capítulo 3: Ontologia de Colaboração. Em Pimentel $\&$ Fuks (org). Sistemas Colaborativos. Elsevier. 